\title{
Petrous Ridge Meningioma Detection in a Patient Treated for Cancer Tongue
}

\author{
${ }^{1}$ Mohammad Waheed El-Anwar, ${ }^{2}$ Ezzedden Elsheikh, ${ }^{3}$ Ismail Elnashar
}

\begin{abstract}
We report a previously unreported case of a 59-year-old ex-smoker male with association between tongue squamous cell carcinoma (SCC) and petrous ridge meningioma. Tongue SCC was successfully treated by partial glossectomy with left functional neck dissection, 6 months later, petrous ridge meningioma was discovered and gamma knife treatment was undertaken for it.
\end{abstract}

Keywords: Cancer tongue, Meningioma, Petrous ridge.

How to cite this article: El-Anwar MW, Elsheikh E, Elnashar I. Petrous Ridge Meningioma Detection in a Patient Treated for Cancer Tongue. Int J Otorhinolaryngol Clin 2014;6(3):127-129.

Source of support: Nil

Conflict of interest: None

\section{INTRODUCTION}

Squamous cell carcinoma (SCC) represents 90 to $95 \%$ of all malignant neoplasms of the oral cavity, being located mainly in the tongue, especially in the lateral posterior border. It generally affects men aged over 50 , most of them with a history of high tobacco and alcohol consumption. ${ }^{1}$

Meningioma is regarded as benign vascular tumor that arises from arachnoid cap cells of the dura mater surrounding the brain and spinal cord. They account for approximately $20 \%$ of all intracranial tumors in adults. It occurs spontaneously or following exposure to cranial low-dose radiation. ${ }^{2}$

Meningiomas are generally benign, slow growing tumors that may produce neurological symptoms and signs due to their compression of adjacent structures. ${ }^{3}$

Only one case showing both meningioma and tongue cancer had previously been reported in the literature. ${ }^{4} \mathrm{We}$ report a case of petrous ridge meningioma subsequently diagnosed in a patient with early tongue carcinoma treated by partial glossectomy.

\footnotetext{
${ }^{1,3}$ Assistant Professor, ${ }^{2}$ Professor

${ }^{1-3}$ Department of Otorhinolaryngology and Head and Neck Surgery, Faculty of Medicine, Zagazig University, Egypt
}

Corresponding Author: Mohammad Waheed El-Anwar Assistant Professor, Department of Otorhinolaryngology and Head and Neck Surgery, Faculty of Medicine, Zagazig University Egypt, Phone: 0020552287298, e-mail: mwenteg@yahoo.com

\section{CASE REPORT}

An ex-smoker male aged 59 years with no history of previous malignancy or radiotherapy was presented with left marginal tongue ulcer (involving the lateral border of the anterior 2/3) (Figs 1A to D). A punch biopsy that was taken from the edge proved histopathologically to be infiltrative SCC grade II. Clinical neck examination could not detect any palpable lymph node enlargement and the patient was staged as T1N0M0.

Patient was prepared and admitted for left partial glossectomy (Figs 1A to D) with left functional neck dissection. Postoperative pathological examination confirmed the tongue ulcer to be SCC with all margins free of malignancy and lymph nodes had no metastatic deposits (0/36) so, apart from follow-up, no additional therapy was needed.

Postoperative period was uneventful and the patient was discharged with no further complaint.

Follow-up of the patient showed apparently normal and healed mucosa without ulceration or recurrence so far (9 months) (Figs 1A to D).

During routine follow-up after 6 months, the patient complained of persistent left deep headache for which neurosurgical consultation was requested and magnetic resonance imaging (MRI) was ordered and showed $3.1 \mathrm{~cm}$ sessile broad base mass on the left petrous ridge. It was isointense on T1-weighted images and hyperintense on T2-weighted images with a dural tail sign and a right convexity compressing the parietal lobe and associated with cerebral edema which was diagnosed as petrous ridge meningioma (Figs 2A and B). Audiometry showed normal hearing bilaterally. Patient was accepted for gamma knife treatment that was performed with 12 Gy to the $50 \%$ isodose and $99 \%$ cover. The patient is still under follow-up in a good health with no more complains.

\section{DISCUSSION}

With the advent of MR technology, the quality of diagnostic imaging for meningiomas has improved dramatically. Magnetic resonance imaging provides improved delineation of dura and sinus involvement, and even information about a tumor's consistency. ${ }^{5} \mathrm{~A}$ characteristic and highly specific feature of meningiomas is the 'dural tail sign' surrounding the dural perimeter of the mass ${ }^{5-7}$ that was reported in MRI of our case. 

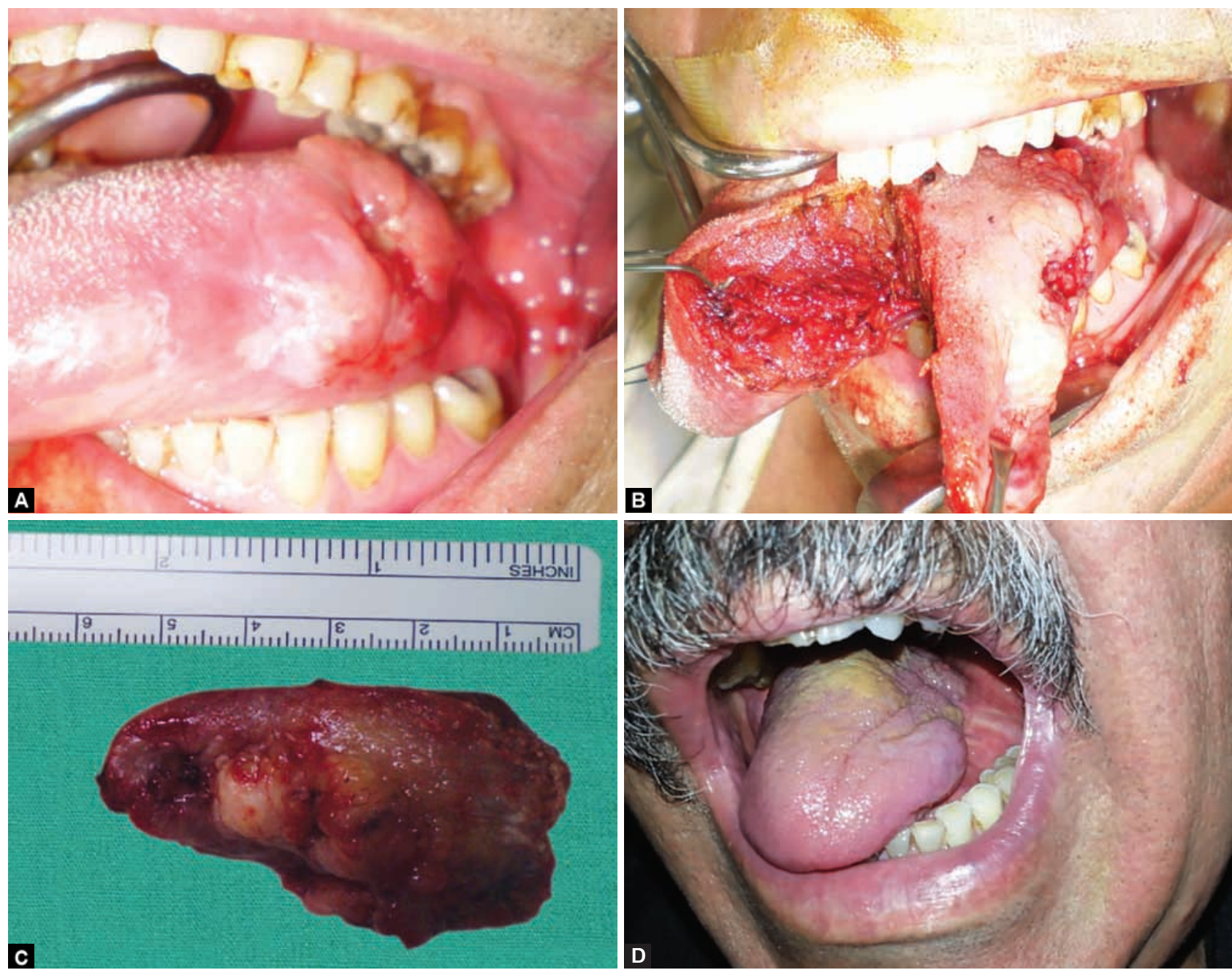

Figs 1A to D: (A) Malignant tongue ulcer, (B) partial glossectomy, (C) removed specimen and (D) healed tongue ( 9 months postoperative)
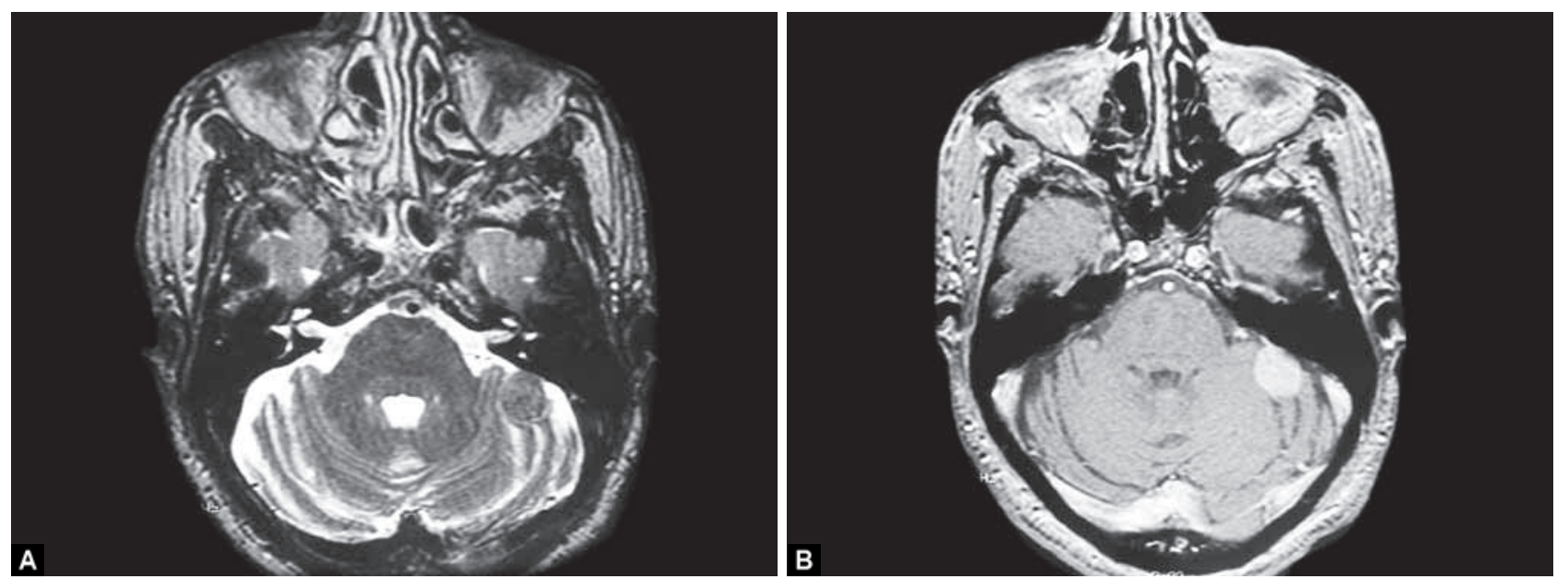

Figs 2A and B: (A) Axial T1-weighted MRI showing isointense rounded petrous ridge meningioma and (B) enhanced axial T1-weighted MRI showing enhanced rounded petrous ridge meningioma with dural tail enhancement axial flair MRI image showing mass to be hyperintense and focally compressing cerebral cortex

We reported a unique case with petrous ridge meningioma after partial glossectomy for early tongue cancer (SCC). Only one previous case had been reported to have meningioma in patients with tongue cancer ${ }^{4}$ but it was asymptomatic frontal meningioma detected incidentally at MRI during the preoperative assessment of the tongue cancer in an elderly male aged 78 years.

The case we described started to complain of brain mass about 6 months after tumor resection, and it is known that brain metastases typically develop 6 months 
to 2 years after diagnosis ${ }^{8}$ so, it should be considered in the differential diagnosis. Brain metastases are among the most feared complications of cancer as they cause profound neurologic symptoms that severely impair quality of life and are associated with poor prognosis. ${ }^{9}$ Most metastases from SCC are reported to occur in liver, lungs and bones; therefore preoperative tumor staging is focused on these sites. ${ }^{10}$ But, distant metastases from oral SCC are extremely rare, to date only few cases have been reported previously, ${ }_{1}^{11}$ it is usually expected in cases of advanced locoregional disease and typically located in the cortical/subcortical areas. Magnetic resonance imaging usually shows metastases to be spherical and well demarcated from the adjacent brain tissue, it is hyperintense on T1-weighted images after gadolinium injection, T2-weighted images and flair sequences demonstrate the surrounding edema and in 65 to $80 \%$ they are multiple. ${ }^{8}$

Because, the reported case showed early stage tongue cancer, the site of the brain mass and the MRI characteristics are suggestive of meningioma, the diagnosis of metastases was excluded.

The clinical management of patients with meningiomas had changed over the past decade because of improved diagnostic imaging, better results with surgery and interventional neuroradiology, and the advent of radiosurgery. ${ }^{6}$

Gamma knife radiosurgery (GKRS) for small and medium sized meningiomas has proved to be an effective alternative to microsurgical resection, radiotherapy, and Linac-based radiosurgery for adjunctive and primary treatment of selected patients with basal meningiomas. ${ }^{6}$ Our case had small, spherical lesion that was an ideal target for GKRS. Radiosurgery for meningioma diagnosed by imaging without tissue diagnosis is associated with a high rate of tumor control and acceptable morbidity but carries a small risk of incorrect diagnosis. ${ }^{7}$

To the best of the authors' knowledge, this case report is the first regarding the successful treatment of both tongue cancer and petrous ridge meningioma in the same patient.

Neck dissection is commonly performed in the management of head and neck malignancy and may involve internal jugular vein (IJV) sacrifice. Intracranial hypertension as a potential complication is well-documented after bilateral neck dissection and reported after unilateral IJV sacrifice. ${ }^{12}$ Every effort should be done to preserve the IJV in neck dissection to avoid the potential risk of intracranial hypertension, which will add more risk and morbidly in the presence of any coincidental intracranial lesion.

\section{CONCLUSION}

Meningioma should be considered in the differential diagnosis of any brain mass associated with tongue cancer and radiosurgery is a good tool in its management.

\section{REFERENCES}

1. Hirota SK, Migliari DA, Sugaya NN. Oral squamous cell carcinoma in a young patient-case report and literature review. An Bras Dermatol 2006;81(3):251-254.

2. Goshen Y, Stark B, Kornreich L, Michowiz S, Feinmesser M, Yaniv I. High incidence of meningioma in cranial irradiated survivors of childhood acute lymphoblastic leukemia pediatr blood cancer. Pediatr Blood Cancer 2007 Sep;49(3):294-297.

3. Backer-Grøndahl T, Moen BH, Torp ST. The histopathological spectrum of human meningiomas. Int J Clin Exp Pathol 2012; 5(3):231-242.

4. Sano K, Kitai R, Yoshimura H, Ohba S. Frontal meningioma incidentally detected in the routine preoperative assessment of tongue cancer: a case report and literature review. J Oral Maxillofac Surg 2013;71(12):2195.

5. Engelhard HH. Progress in the diagnosis and treatment of patients with meningiomas-Part I: diagnostic imaging, preoperative embolization. Surg Neurol 2001;55(2):89-101.

6. Kreil W, Luggin J, Fuchs I, Weigl V, Eustacchio S, Papaefthymiou JG. Long-term experience of gamma knife radiosurgery for benign skull base meningiomas. Neurol Neurosurg Psychiatry 2005;76(10):1425-1430.

7. Flickinger JC, Kondziolka D, Maitz AH, Lunsford LD. Gamma knife radiosurgery of imaging-diagnosed intracranial meningioma. Int J Radiat Oncol Biol Phys 2003;56(3):801-806.

8. Westphal $\mathrm{M}$, Heese $\mathrm{O}$, Dewit M. Intracranial metastases: therapeutic options. Annals of Oncology 2003;(suppl 3): I4-10.

9. Lassman A, DeAngelis L. Brain metastases. Neurol Cin 2003;21:1-23.

10. Calhoun K, Fulmer P, Weiss R, Hokanson J. Distant metastases from head and neck squamous cell carcinoma. Laryngoscope 1994;104(10):1199-1205.

11. Hardee P, Hutchinson I. Intracranial metastases from oral squamous cell carcinoma. Br J Oral Maxillofac Surg 2001;39(4):282-285.

12. Ward MJ, Faris C, Upile T, Patel NN. Ophthalmoplegia secondary to raised intracranial pressure after unilateral neck dissection with internal jugular vein sacrifice. Head Neck 2011;33(4):587-590. 